\title{
Application of Nanomaterials in Stem Cell Regenerative Medicine of Orthopedic Surgery
}

\author{
Su Pan, ${ }^{1,2}$ Hongmei Yu, ${ }^{3}$ Xiaoyu Yang, ${ }^{1}$ Xiaohong Yang, ${ }^{2}$ Yan Wang, \\ Qinyi Liu, ${ }^{1}$ Liliang Jin, ${ }^{4}$ and Yudan Yang ${ }^{3,5}$ \\ ${ }^{1}$ Department of Orthopedics, The Second Hospital of Jilin University, Changchun, Jilin, China \\ ${ }^{2}$ Jilin University School of Pharmaceutical Sciences, Changchun, Jilin, China \\ ${ }^{3}$ China-Japan Union Hospital of Jilin University, Changchun, Jilin, China \\ ${ }^{4}$ Department of Pathobiological Sciences, Louisiana State University, Baton Rouge, LA, USA \\ ${ }^{5}$ State Key Laboratory on Integrated Optoelectronics, College of Electronic Science and Engineering, Jilin University, \\ Changchun, Jilin, China
}

Correspondence should be addressed to Yudan Yang; yangyudan@jlu.edu.cn

Received 27 January 2017; Accepted 8 March 2017; Published 6 July 2017

Academic Editor: Jian Zhong

Copyright (C) $2017 \mathrm{Su}$ Pan et al. This is an open access article distributed under the Creative Commons Attribution License, which permits unrestricted use, distribution, and reproduction in any medium, provided the original work is properly cited.

Regenerative medicine aims to achieve functional rehabilitation of tissue or cells injured through wound, disease, or aging. Recent findings suggest that nanotechnology provides advanced biomaterials with specified morphologies which can create a nanoscale extracellular environment capable of promoting the adhesion and proliferation of stem cells and accelerating stem cell differentiation in a controlled manner in tissue engineering. This review summarizes the biological effects of nanomaterials and their regenerative medicine applications in orthopedic surgery research, including bone, cartilage, tendons, and nerve tissue engineering.

\section{Introduction}

Stem cell is a special class of cells which can self-renew and differentiate into specific types of functional cell (such as cardiac cells, pancreatic islet cells, and nerve cells), and then these cells are transplanted into the patient to replace damaged tissue, and it is the basic principle of regenerative medicine. Stem cells and regenerative medicine is a new biomedical field in recent years with significantly clinical value, which promotes wound healing of body and can be used in treatment of disease through stem cell transplantation, differentiation, and tissue regeneration. With more and more trauma and increasing aging population, an incremental number of orthopedic procedures are performed with orthopedic implants annually. However, there are many complications for traditional orthopedic implants, such as infection, poor host tissue conformity, and implant loosening resulting in treatment failure.

Nowadays, nanotechnology and nanomaterials are heavily employed in various biomedical fields [1-11]. Among them, regeneration therapy using stem cells and nanometer technology both belong to the latest conduits of biotechnological research. One of the main objectives for a successful implementation of regenerative medicine treatment is to control the preservation and proliferation of stem cells and to accelerate their differentiation in a managed manner. The development of nanometer technology has provided an access for unambiguous comprehension of stem cell therapy in vivo by mimicking the environments of extracellular matrix in the culture, and nanotechnology seems to adapt a great possibility in providing new outlook for stem cell research. Nanotechnology carries in its innovation of dynamic three-dimensional nanoenvironments or nanoscaffolds with patterned nanomorphologies and different bioactive molecular substrates for preservation, proliferation, and differentiation of stem cells required for advancement of tissue engineering. Novel regenerative medicine methods combine different scaffold biomaterials with stem cells to provide biological implant or substitutes that can repair and eventually improve tissue functions. 


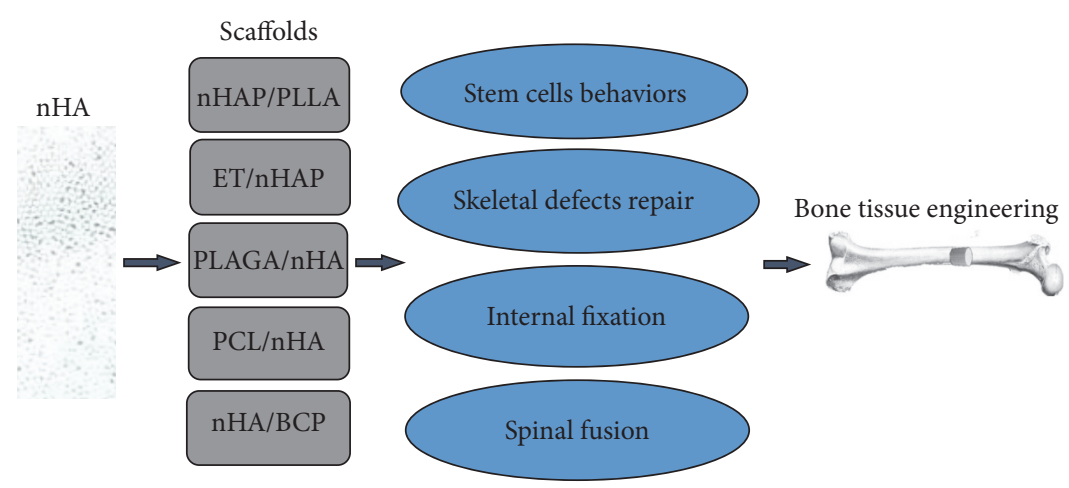

FIGURE 1: The application of nHA scaffolds in bone tissue engineering.

This review will discuss the biological effects of nanomaterials and their regenerative medicine applications in orthopedic surgery research, including bone, cartilage, tendons tissue, and nerve tissue engineering.

\section{Bone Tissue Engineering}

Currently, bone compositions fabricated according to the fundamental of tissue engineering are being regarded as an ideal selection for the functional restoration of segmental deossification. Biomaterials used for bone tissue engineering include nanohydroxyapatite, titanium, calcium phosphate, graphene oxide, carbon nanotubes, or the compound of them [12-14]. The surfaces or three-dimensional nanostructures of implant which better mimic the naturalistic environments of bone extracellular matrix in the culture, naturally nanocompound tissue, can promote the osteogenic differentiation of stem cell which is important for bone tissue engineering.

2.1. Nanohydroxyapatite. Hydroxyapatite is the principal inorganic mineral component of animal and human teeth and bones which is difficult to be dissolved in a solution and to be utilized in various applications. If nanometer size hydroxyapatite (nHA) particles can be made, it will exhibit a range of unique properties (Figure 1).

2.1.1. Effect on Biological Behavior of Stem Cells. Three different shapes of nHAP/PLLA scaffolds were prepared, such as needle structure, spherical structure, and rod structure, on which passaged-3 rat MSCs were cultured in threedimensional scaffolds, and it was demonstrated that needle nHAP/PLLA scaffolds appeared to provide the most proportionate environment for bone regeneration using MSCs. It was demonstrated that a novelly fabricated eri-tasar silk fibroin nanofibrous scaffold (ET) scaffold hybrided with nHA deposition by a surface precipitation method stimulated osteogenic differentiation in the absence of any growth factors due to the improved properties of it in physical chemistry and biology. It may offer effectual and profitable biomaterials for bone tissue engineering in orthopedic surgery by promoting stem cell differentiation on ET/nHAP scaffold without any growth factor additive in stem cell culture [15]. A fibrous structure and synergistic action of collagen and nHA with high molecular weight poly-L-lactide (PLLA) played a crucial role in stimulating human mesenchymal stem cells (hMSCs) differentiation towards osteogenic lineages, indicating its possibility for bone defect repair based on stem cell therapies [16].

Conventional biphasic calcium phosphate (BCP) ceramics with porous structure, displaying superior bone conduction and biocompatibility without intrinsically osteoinductive, were coated with nHA due to supernal osteoinductive potentiality of it. The new nHA coating BCP scaffolds were demonstrated to be more suitable for the adhesion and proliferation of stem cell and MSCs osteogenic differentiation as compared with the conventional BCP scaffolds, indicating the property of nHA conducive for osteoinduction making this scaffolds more adaptive for applications in bone regeneration [17]. The proliferation, osteogenic phenotypic markers expression, and mineral deposition were elevated when hMSCs were seeded on a new three-dimensional, porous poly (D,L-lactide-co-glycolide) (PLAGA)/nHA scaffold suitable for high-aspect ratio vessel (HARV) bioreactor applications, and the degradation pattern of the scaffold was not altered by nHA incorporation and maintained its mechanical integrity for 6 weeks in the dynamic culture environment, which can be used as a potential tissue engineering matrix allowing for the generation of bone tissue [18]. Upon culturing hMSCs on a bioactive nanofiber scaffold made of poly-caprolactone (PCL) and nHA, it was demonstrated that the mineral phase existence increased alkaline phosphatase (ALP) activity and mRNA expression levels of genes related to osteoblast without any osteogenous composition, and it was suggested that the architecture of this novel nanofibrous scaffolds and its chemical constituent could regulate hMSCs osteogenic differentiation [19]. In conclusion, nanofibrous scaffolds composed of nHA manufactured to different shape or hybrided with various nanomaterials will have favourable effect on biological behavior of stem cells and then enhance osteoinductive potential of it by some molecular biology mechanism.

2.1.2. Skeletal Defects Repair. Successful clinical repair of nonhealing bone defects relies on artificial bone with robust efficiency of osteoinduction and outstanding biomechanical stability. Allogenic BMSCs seeded on the nHA/polyamide 
6 (nHA/PA6) composite scaffolds used as porous matrices were implanted in the $8 \mathrm{~mm}$ diameter calvarial defect of rats, and this showed good biocompatibility and osteoinduction which enhanced bone inductivity at the initial stage after implantation [20]. A composite scaffold composed of nHA-type I collagen beads, BMSCs, and platelet rich plasma (PRP) enhanced significantly the new bone formation which could generate transplants with effectively meliorative bone tissue reorganization promising skeletal defects restoration [21]. It was used to repair successfully the nodulated skeletal defectiveness by eight weeks after operation of novel $\mathrm{nHA} /$ collagen/PLLA/chitin fibers (nHACP/CF) scaffolds with goat bone mesenchymal stem cells (BMSCs) cultured and autograft bone. Furthermore, the results revealed that the level of ALP and DNA in goat BMSCs cultured on it was markedly increased. It was demonstrated that this novel scaffold could be a proper graft for bone defects repair [22]. In conclusion, in cases of large bone defects resulting from trauma, infection, tumors, osteomyelitis surgical debridement, and a variety of congenital diseases, tissue-engineered structures may provide alternatives to traditional bone substitute by culturing autogeneic stem cells of patients with nHA composite scaffolds in culture system.

2.1.3. Internal Fixation. Bone plate set and screws for bone fractures therapy by internal fixation methods showed excellent biocompatibility and biomechanical properties. The nHA/polyamide 66/glass fiber compound with good biocompatibility and biomechanical properties was demonstrated to enhance the attachment and proliferation of MSCs without any negative effect on the mineralization of matrix and MSCs osteogenesis. This new compound might be used for operations by internal fixation [23].

2.1.4. Spinal Fusion. Spinal fusion surgery performed with an autologous osseous graft obtained from the crest of ilium is a conventional treatment in therapy spondylolisthesis in children or lumbago derived from intervertebral discs degeneration. However, the procedure is foregone to have several shortcomings, such as nearthrosis, ache, or the adverse reactions of blood transfusion. It has been demonstrated that a novel graft material comprised of a new matrix of mineralized collagen and nHA integrated with autogeneic adipose-derived mesenchymal stem cells (ADMSCs) had effective impact on rabbit posterolateral spinal fusion [24].

In conclusion, nanohydroxyapatite scaffold has both nanomaterials properties and good biocompatibility which render it a very broad application prospect in bone regenerative medicine, including the regulation of stem cell biological behavior (such as proliferation and differentiation), the improvement of bone tissue regeneration promising skeletal defects repair, internal fixation surgery, and spinal fusion in orthopedic surgery.

2.2. Ti. Osteogenic cells belong to anchorage-dependent cells in which the increase of adhesion and cytoskeleton changes in early stage is imperative to stimulate osteogenesis and to accomplish a more expedient osseointegration of implant materials surfaces in vivo. Metallic implant materials including titanium and its alloys are conventionally used in orthopedic applications due to their superior mechanical functions and biological unreactiveness.

2.2.1. Effect of Topography and Measurement. It was presumed that the original progenitor cells morphology on the nanoscale surface topography might have effect on the succedent stem cell functions [25].

Stem cells were cultured onto titanium discs with different structures, such as sandblasted, sandblasted and large-grit acid etched, and full contact coverage (FCC). The results showed that the FCC titanium surface on which osteoblastlike cells matured most rapidly might have a crucial effect on the primeval phase of bone union course [26]. Different micro- and nanostructure of a new nanosized oxidized titanium graft enhanced the adhesion and osteogenic differentiation of BMSC plated onto the oxidative surfaces which exhibited a greater tanglesome micro- and nanosized structures and had increased levels of coarseness parameters contrasting to the convention alone [27]. Titanium surface features were controlled as subnano-, nano-, and submicron scales which could activate integrin-ligand proteins interactions, and then the results showed that osteoblast differentiation of primary mouse BMSC was accelerated significantly only on the surface of titanium with nano- and submicron hybrid after 2 weeks. Furthermore rapid cytoskeletal reorganization of BMSC on the transparent surface of titanium with nanoscale was demonstrated, which definitively induced increased genes expression level of osteoblast dominant form after three weeks [28]. It was observed that oxidized titanium nanotubes with a diameter of $70 \mathrm{~nm}$ was the optimal dimension for the osteogenic differentiation in adipose-derived stem cells of human (hASCs). Furthermore the results revealed that the nanotopography of oxidized titanium nanotubes directs stem destiny by upregulating methylation level of histone $\mathrm{H} 3$ at lysine 4 in the promoter regions of osteogenic genes Runx2 and osteocalcin, by inhibiting demethylase retinoblastoma binding protein $[15,29]$.

2.2.2. Titanium Surfaces Modification. Human bone ECM is comprised of collagen nanofibers and nanostructured hydroxyapatite particles in nanometer dimension improving cells growth in its nanofibrous porous structure, so the biomimetic and bioactive titanium surface with nanostructured coating might control stem cell adhesion and osseointegration (Figure 2). It was demonstrated that the adhesion and proliferation of osteoblast and MSCs were enhanced on titanium treated with $\mathrm{nHA} /$ carbon nanotubes [30]. The stem cells cultured on titanium nanostructure coated with ECM components exhibited faster osteoblastic differentiation and more efficient deposition of mineralized matrix as compared with uncoated structure. Heatedly oxidative Ti-6Al-4V coating with biomimetic chitosan/alginate film containing nanoscale silver particles enhanced significantly the morphology, viability, and proliferation of BMSC. Furthermore, the composite film restrained the multiplication of Escherichia coli and Staphylococcus aureus and upregulated significantly the expression of ALP [31]. 


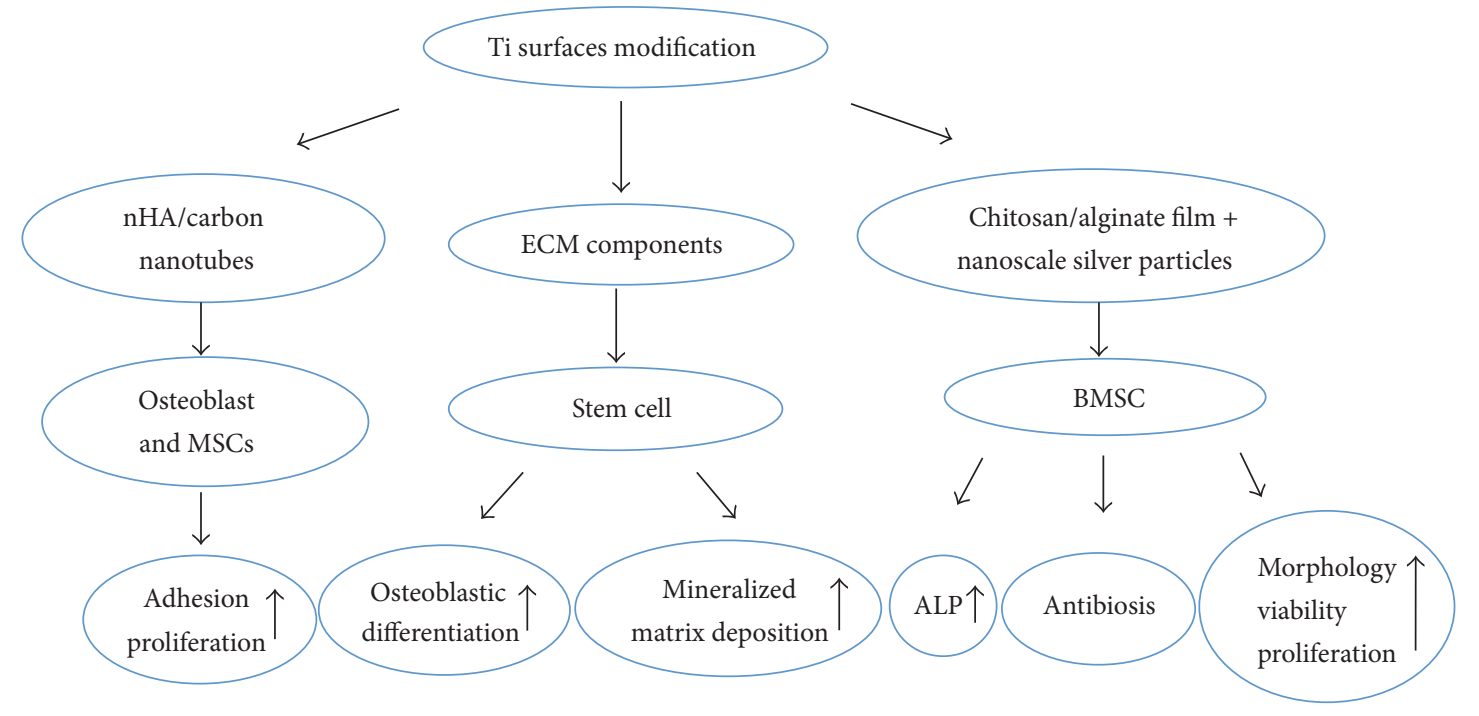

Figure 2: The effect of titanium surfaces modification on stem cell.

Overall, these studies present a towardly stratagem to improve biocompatibility and potential of these novel nanostructured materials for orthopedic applications.

2.3. Calcium Phosphate. Nanostructured calcium phosphate (CaP) biomaterials/scaffolds are of particular interest to meet the increasing need for osteoanagenesis, because they share chemical/crystallographic analogies to inorganic components of bone. It was demonstrated that the MSCs proliferation was enhanced when cultured on the nanosized three methods-biomimetic discs coated with $\mathrm{CaP}$ as compared with the uncoated or galvano-chemically coated structures [32]. Novel porous Cap-Ti6Al4V (Cap-Ti) hybrids with conspicuous physical and chemical properties regulated the propagation, osteogenous differentiation, and matrix mineralization of human periosteum derived cells (hPDCs), such as regulating osteoclast formation by $\mathrm{CaP}$ coating absorption of hPDCs, decreasing gene expression level of osteoprotegerin, and promoting multinucleated giant cells aggregation near to the Cap-Ti hybrids surface. Furthermore, Cap-Ti compound induced heterotopic bone formation during hypodermic implantation by a manner depending on cell density [33].

Calcium phosphate cement (CPC) possesses superior osteoconductivity and it is easy to be assimilated or replaced by regenerative bone tissue, so CPC is suitable to be injected in bone defect position to form powered supports. It was demonstrated that umbilical cord mesenchymal stem cells of human (hUCMSCs) attaching to high-strength CPC showed superior propagation or osteogenous differentiation. The results revealed that hUCMSCs transported by CPC might be a probable alternative in orthopedic treatment [34]. However, CPC is limited to non-stress-bearing repairs due to the low strength of it that can be reinforced by chitosan incorporation. It was reported that MSCs differentiated to osteoblasts cell lines and expressed ALP in high level which is a bone marker on the CPC and chitosan scaffolds with increased strength as compared to CPC [35]. Magnesium phosphate cement (MPC) was combined with CPC to develop novel calcium-magnesium phosphate cement (CMPC) which was implanted into bone defects in rabbits. It was demonstrated that CMPC has a shorter setting time and markedly better mechanical properties than either CPC or MPC. Furthermore, the histological evaluation showed that the introduction of MPC into CPC enhanced the efficiency of new bone formation, and the CMPC also exhibited good biocompatibility, biodegradability, and osteoconductivity with host bone in vivo [35].

2.4. Graphene. Polymeric material composed of graphene is developed for biomedical applications, such as biosensing for the increased electrical conductivity of the graphene composite material. Furthermore, soft biomaterials can be reinforced by graphene incorporated to polymer. Graphene oxide (GO) is a broadly investigated form of grapheme. $\mathrm{GO}$ is rich in oxygen, including functional groups, such as epoxide carboxyl and hydroxyl groups. It is important for bone regeneration therapy to control the propagation and differentiation of stem cells in a controlled manner. It was proved that osteogenic differentiation of stem cell is increased when cultured on mechanically stiff substrates. Graphene could provide a towardly biocompatible scaffold to accelerate the specific differentiation of hMSCs into bone cells without hampering their proliferation [36]. GO flakes conjugated to collagen sponges which were clinically approved scaffolds to provide soft microenvironment for bone regeneration increased the scaffold stiffness 3-fold without cytotoxicity and enhanced significantly osteogenic differentiation of hMSCs, so this novel 3D GO-collagen scaffolds could offer a novel platform for stem cell study and bone regeneration [37]. PCL compound of $\mathrm{GO}$, reduced $\mathrm{GO}$ ( $\mathrm{RGO}$ ), and amine-functionalized GO (AGO) were prepared at various filling material components. The results demonstrated that AGO and GO particles significantly enhanced hMSCs proliferation, and AGO was most effectual in modulating the osteogenic differentiation of stem cell leading to mineralization [38]. 


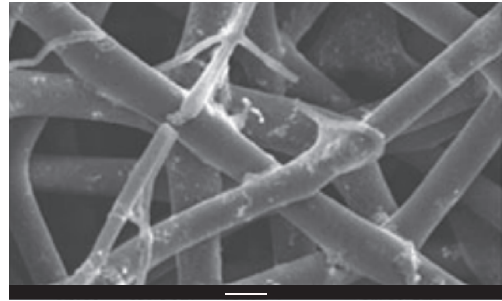

$15 \mathrm{kV} \quad \times 1,000 \quad 10 \mu \mathrm{m} \quad 10 \quad 40 \quad$ SEI

(a)

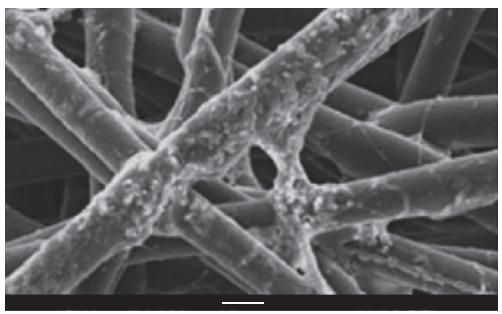

$15 \mathrm{kV} \quad \times 1,000 \quad 10 \mu \mathrm{m} \quad 11 \quad 36 \quad$ SEI

(d)

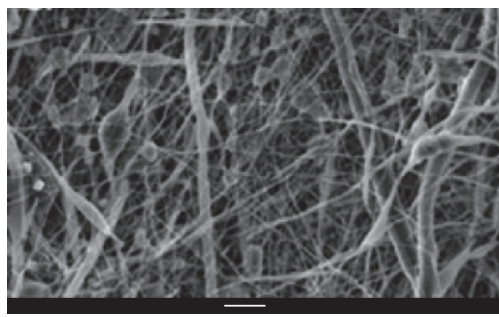

$15 \mathrm{kV} \quad \times 1,000 \quad 10 \mu \mathrm{m} \quad 11 \quad 36 \quad$ SEI

(g)

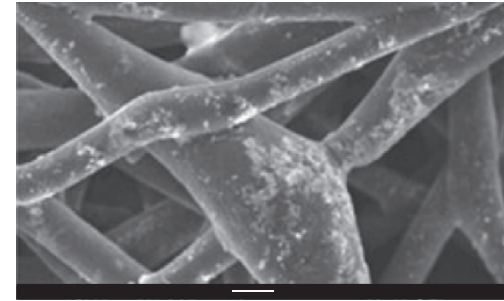

$15 \mathrm{kV} \quad \times 1,000 \quad 10 \mu \mathrm{m} \quad 10 \quad 40 \quad$ SEI

(b)

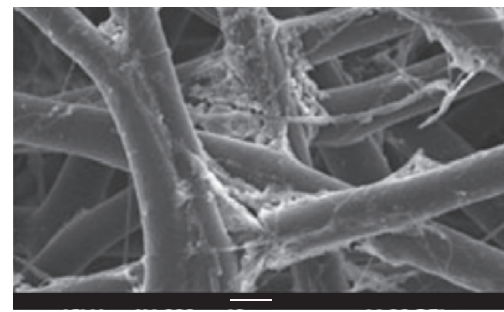

$15 \mathrm{kV} \quad \times 1,000 \quad 10 \mu \mathrm{m} \quad 11 \quad 36 \quad$ SEI

(e)

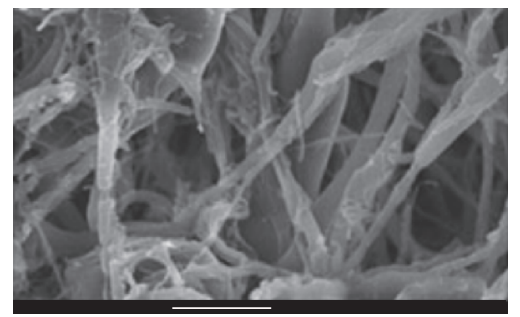

$15 \mathrm{kV} \quad \times 2,500 \quad 10 \mu \mathrm{m} \quad 10 \quad 36 \quad$ SEI

(h)

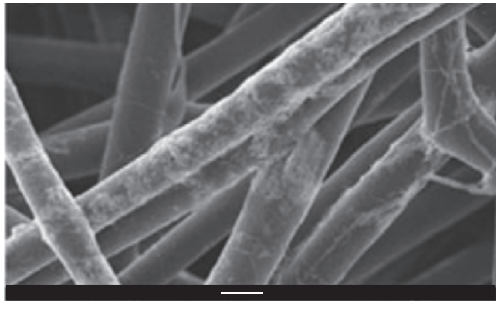

$15 \mathrm{kV} \quad \times 1,000 \quad 10 \mu \mathrm{m} \quad 11 \quad 36 \quad$ SEI

(c)

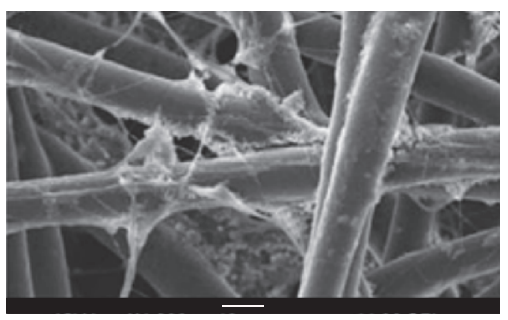

$15 \mathrm{kV} \quad \times 1,000 \quad 10 \mu \mathrm{m} \quad 11 \quad 36 \quad$ SEI

(f)

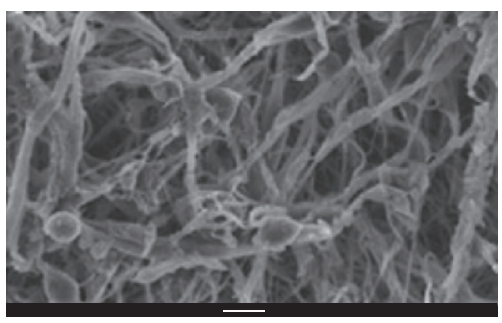

$15 \mathrm{kV} \quad \times 1,000 \quad 10 \mu \mathrm{m} \quad 10 \quad 36 \quad$ SEI

(i)

FIGURE 3: SEM image showing biomineralization in untreated (a, d, g), argon-treated $(b, e, h)$, and nitrogen-treated $(c, f, i)$ microfibers $(a-c)$, micro/nanofibers (d-f), and nanofibers (g-i). Figure 3 is from [40].

2.5. Topography of Nanomaterial Surface. One important goal in tissue engineering is to understand and control the factors directing cell proliferation and differentiation into a special cell lineage in the nanoscale environment, and it is a useful method to control cell biological behaviors by material surface. It was demonstrated that the adhesion and differentiation of MSC were enhanced by ion beam irradiation $(10 \mathrm{keV} \mathrm{He}+)$ which induced negligible surface smoothening and various nanostructured surface of PCL [39]. Microhybrid, micro/nanohybrid, and nano-PCL fibers were subjected to plasma treatment with argon and nitrogen in low pressure, by which PCL scaffold with superhydrophobicity converted to hydrophilic scaffolds with modified biomineralization (Figure 3). Furthermore, it was demonstrated that cell spreading, elongation, attachment, proliferation, and protein adsorption were increased remarkably on microand micro/nanofibers with plasma treatment, cell attachment was enhanced significantly on plasma-treated nanofibers, and differentiation towards osteoblast lineage was accelerated in fibers with plasma treatment [40].

The biological behavior of stem cells can be controlled by physical factors such as the micro- and nanotopography of the extracellular matrix. It was demonstrated that nanofibrous combinatorial scaffolds can influence the fate of human BMSC without chemical treatments or cellular reprogramming by mimicking the extracellular matrix topographical environment [43]. Changing the depth of biomaterial surface structures enhanced hMSCs adhesion and induced specific differentiation into osteoblast-like cells without osteogenic medium which was dictated by stress through focal-adhesion-induced restructuring of F-actin filaments [44]. Another investigation demonstrated that different forms of biomaterials control osteogenic differentiation and growth of stem cell by differential activation focal adhesion kinase [45].

These studies have fundamental implication for comprehension of the architectural effects of extracellular matrix microenvironment and also for the regenerative medicine of orthopedic surgery using stem cells.

\section{Cartilage Tissue Engineering}

Nanostructured cartilage tissue is traditionally difficult to regenerate because of its extremely poor inherent regenerative capacity and perplexing hierarchical structures. Traditionally medical treatments are not noninvasive and may 
cause many complications. Nanomaterials fabrication committed to producing useful biologically scaffolds of tissueengineered cartilage used to promote the chondrification of stem cells. Several novel 3D biomimetic nanostructured scaffolds were studied which was fabricated by PLLA polymers and multiwalled carbon nanotubes treated with hydrogen. The scaffolds embedded by multiwalled carbon nanotubes exhibited a vigorous enhancement in shatter strength with a compressed Young's modulus accordant to human cartilage. It was demonstrated that MSCs was preferred to attach on smaller fiber diameter structure and increasing chondrogenic differentiations were induced by combination of plasmatreated multiwalled carbon nanotubes and PLLA [46].

Another investigation demonstrated that the attachment, distribution, viability, and propagation of stem cells cultured in vitro in the $3 \mathrm{D}$ nHA coated poly(lactide-co-glycolide) scaffolds were increased as compared with poly(lactideco-glycolide) one. Furthermore, it was demonstrated that the osteochondral defects of rats knees treated by threedimensional poly(lactide-co-glycolide)/nHA scaffold combining with MSCs were stuffed with crystalline cartilage. Furthermore, there was affluent mucopolysaccharide and collagen type II sedimentation after surgery. The results suggested that the poly(lactide-co-glycolide)/nHA-MSCs compound might have probable utilization in tissue-engineered cartilage [47]. Traditionally, chondrogenic differentiation is accomplished by stem cells culturing with chondrogenic factors in the culture medium, such as the protein transforming growth factor-beta 3. However, cell-to-cell interaction and diffusional limitation of transforming growth factor-beta 3 mainly provided by pellets may restrain stem cells differentiation to restrain. It was demonstrated that a novel GO could be used as substrates for adhesion of stem cells and delivered growth factor during the stem cell differentiation to cartilage. GO used to adsorb fibronectin and transforming growth factor-beta 3 was incorporated in pellets of hASCs to obtain a hybrid pellets of hASC-GO sheets ( size $=0.5-1 \mathrm{mu} \mathrm{m}$ ) which promoted the hASCs differentiation to cartilage through increasing the interaction of cell with fibronectin and providing transforming growth factor-beta 3 efficiently. The novel hASC-GO sheets may offer novel methods for hASC culture to obtain tissue-engineered cartilage [48].

\section{Tendons Tissue Engineering}

The reconstructive articulation usually tends to fail at the tendon-to-bone insertion site, so an orthobiologic material is formidably required to provide a stable transition from hard bone to soft ligament tissue.

A nanostructured material using PLLA mineralized with hydroxyapatite and magnesium nanoparticles was developed to regenerate the tendon-to-bone insertion site which was fashioned in the shape of an o-ring to recover functionality to injured entheses, the graded transition of mineralized fibrous cartilage connecting ligament to bone. Tendon stem cells are useful in effectual restoration or regeneration of wounded tendons. The destiny of tendon stem cells is not unambiguous during the implantation. It was demonstrated that tagging tendon stem cells with super-paramagnetic iron oxide (SPIO) nanomaterials was a practicable method for tracing tendon stem cells used to determine the feasibility of it. This novel SPIO labeled tendon stem cells provide a noninvasive approach to wounded tendons observe restoration [49].

\section{Nerve Tissue Engineering}

Spinal cord injury is defined as the direct or indirect factors that cause spinal cord damage, in the corresponding segment of the injury, associated with motor, sensory and sphincter dysfunction, muscle tension abnormalities and pathological changes, and so on. The extent and clinical manifestations of spinal cord injury depend on the location and nature of the primary injury. Spinal cord injury is the most serious complication of spinal injury, which often leads to severe dysfunction of the injured segment following limb. Infection, tumor, spinal degenerative disease, scoliosis, myeloschisis, spondylolisthesis, and various types of spinal fractures and dislocations caused by indirect external forces can lead to spinal cord injury. The nerve repair of spinal cord injury is a difficult and important problem in regenerative medicine, and more and more research is used to repair the nerve along with the depth of people's understanding in the nerve biology and material science.

5.1. Effect of Surface Topography. Conclusive extracellular environment has a crucial impact in cell life by regulation of cell morphology, proliferation, survival, and differentiation into a specific cell line. A main restraint in the clinical application of stem cell technique is the lack of available regulate of its biological behavior, such as attachment, multiplication, and differentiation. Current in vitro studies have indicated that biological materials with nanosurfaces morphology can affect cell behavior. Thus, confirmation of biological materials supporting adequate ES cell adhesion, proliferation, and differentiation into special cell linage is a fascinating measure to investigate. Current medicinal cell therapy, using the special ability of stem cells in differentiating to nerve corpuscles which is loaded by polymer materials, focuses on the restoration of the central nervous system damage.

A thin film of gold with different roughness of surface morphology was fabricated by the conjugation of microfabrication technology. Then the biomimetic possibility of the thin film was investigated, compared with glass coverslips and plastic tissue culture materials, for regulating the differentiation of neural precursor derived from ES cell. It was demonstrated that the neural precursors derived from ES cells which were cultured for five days had the best adhesion on the gold films and were carried out with the maximum differentiation on gold films with surface roughness of standard deviation $21 \mathrm{~nm}$ without any conventional diffluent neurotrophic factor. In addition, the influence of the grating axis on the axons growth direction is observed when the neural precursors derived from ES cell were seeded in a conjunction of microscale trenches and nanoscale surface roughness. These data suggest that biological material design can maximize the differentiation and organization of neural precursors derived from ES cells, and it can be found to be useful as a synergistic supplement of diffluent neurotrophic 


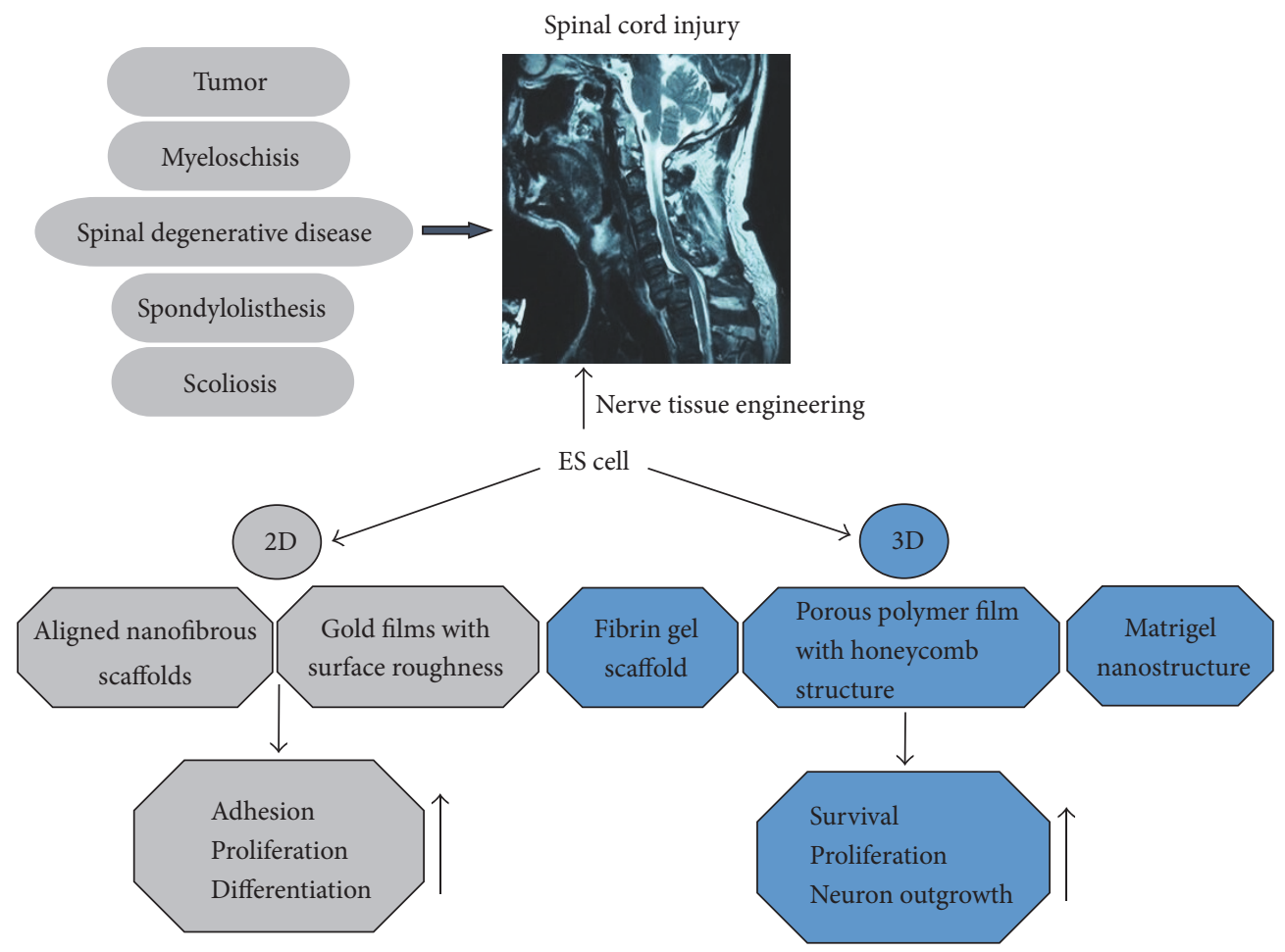

FIgURE 4: The application of stem cell on 2D or 3D scaffold for spinal cord injury.

factor usually used to stimulate and model the differentiation of ES cell in vitro. This basal pattern of gold thin films may be used in the nerve prosthesis with the need for directed induction [50].

The multipotency P19 embryonic cancer cells were cultured on poly(lactic-co-glycolic acid) nanofibrous scaffolds which were fabricated by random and aligned; subsequently it was investigated that the effect of surface morphology of nanofibrous scaffold on the proliferation and retinoic acid induced neural differentiation of P19 cancer cells. It was demonstrated that the surface of aligned nanofibrous scaffolds provided compatible interface for normal cellular functioning and promoted cell proliferation due to their resemblance with naturalistic extracellular matrix [51].

5.2. Effect of Three-Dimensional Scaffold. Nerve tissue engineering belongs to the most towardly approaches to recover the health of the central nervous system damage. Stem cell technique integrated with surface topology of nanofiber scaffolds offers a novel and more effective method for inducing stem cell differentiation and thus to be used for central nervous system functional reconstruction. The goal of $3 \mathrm{D}$ cell culture moulds in vitro is to fill in gaps in the normal $2 \mathrm{D}$ cellular research and the in vivo extracellular matrix (Figure 4). 3D scaffold made up by nanofibers with poriferous structure provides 3D environment for cell distribution, proliferation, and differentiation which has clinical application prospect for tissue engineering. In particular, for nerve historegeneration which is very small regeneration ability, 3D moulds are fundamental in providing support to mimic ECM which is convenient for naturalistic circulation of oxygen, nutriment, and nerve factors and might be beneficial to nerve cell regeneration.

The neuron-like cells derived from human endometrial stem cells (hEnSCs) were cultured in a novel fibrin gel scaffold to control cell behaviors such as survival, proliferation, and neuron outgrowth and to investigate the interactions between cell and matrix. The mechanical characters of the newly prepared fibrin gel scaffolds were investigated which showed the suitable materials, and a good conformity between cell and fibrin gel scaffolds was detected by SEM and TEM. Cell was seeded and cultured on fibrin gel scaffolds for 6 days, and the result showed good expansion and integration of the neuronal network by immunofluorescence assay. Furthermore, the survival, proliferation, and nerve growth of the neuronlike cells were compared between 2D culture and 3D culture, and the results showed that 3D culture has positive influence. These results demonstrated that the novel 3D fibrin gel scaffolds were applied to promote the survival and outgrowth of nerve cell for nerve injury repair [52]. It was demonstrated that neural progenitors (NPs) derived from hEnSCs can express correlative markers such as Pax6, Nidogen, and Soxl and it might experience pluripotency differentiation to neuroglial and neurons. A compositive, random orientation, 3D nanofibrils matrix consisting of polyamine nanofibers prepared by electrospinning methods was adopted to evaluate the behavior of NPs cultured on it. The results showed that the immigration, proliferation, morphology, and neurapophysis length of NPs cell were enhanced when cultured on $3 \mathrm{D}$ scaffolds as compared with 2D structure [53].

The nanostructure scaffold design originated in self organization is the focus of study when looking for novel 
RADA 16-BMHP1

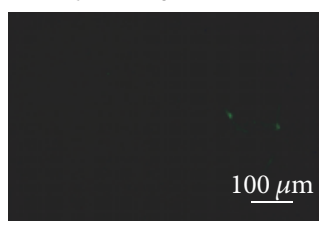

RADA 16-BMHP2

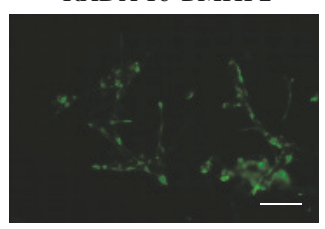

RADA 16-RGD

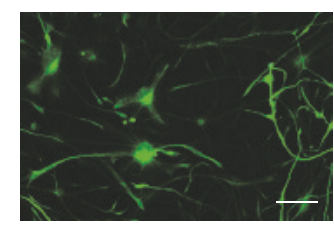

RADA 16

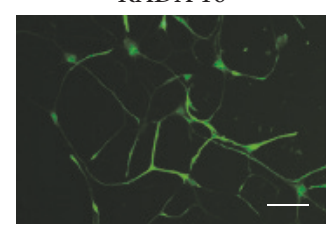

Control

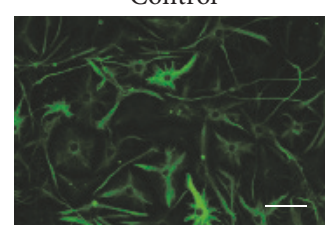

FIGURE 5: The immunofluorescence image of NSCs after 7 days of differentiation. NSCs had been proliferating for 7 days in each of the selfassembling peptides in analysis. Glial fibrillary acidic protein positive cells are shown as control. RADA16, 16-residue peptide; BMHP, bone marrow homing peptide; RGD, Arg-Gly-Asp. Figure 5 is from [41].

biological materials and biosensor, and the new nanostructure scaffold has many kinds of probable utilization for tissue regeneration and iatrical implantation. 3D nanostructures in proportionate aperture and porousness and with coadjacent pores are necessary to promote the agglutination, proliferation, differentiation of stem cells, and ultimate tissue engineering. A new porous polymer film with high regularity in honeycomb structure was fabricated through self-organized formation which had a strong influence in cell biological behavior, such as proliferation, macula adheres, and cell morphology. The results suggested that the biological behavior of neural stem cell and progenitor cells could be affected by honeycomb-patterned polymer film aperture, and this novel honeycomb film is expected to be used for biological material in nerve tissue engineering without any nutrilite in stem cell proliferation. Another novel 3D nanostructured culture system for neural stem cells was fabricated with matrigel biomaterial at different concentrations by self-assembling which possess the formation of a complex and biologically activated matrix displaying exceptionally premeditated serviceable subjects for Arg-Gly-Asp, bone marrow homing peptide 1, and bone marrow homing peptide 2. Neural stem cells were embedded in the 3D scaffolds and detected by microscopy, and the results exhibited that the survival, proliferation, and differentiation of neural stem cells in the 3D nanostructured scaffold were in good condition which indicated that this novel self-assembling 3D matrigel scaffold could be potentially used to create particular premeditated peptides for nerve stem cell lines (Figure 5) [41].

5.3. Effect of Graphene Biomaterial. Although graphene based nanomaterial is still in its infancy, as a novel type of biocompatible biomaterials, it has high performance for biomedical applications in tissue engineering and regenerative medicine because of the good electric conductivity, flexibility, and high molecular absorption of it. In neural tissue engineering and regenerative medicine, research is focused on the exploration of graphene characteristics which can enhance the bioeffect and the application of it on neural cells and stem cells [54].

5.3.1. Two-Dimensional Graphene. It was reported that nanostructure hybrid scaffold of graphene composite material can directionally induce the neural stem cells differentiated into myelinating cells in central nervous system, such as mature oligodendrocytes. In addition, the procedure could be promoted in absence of any differentiation inducing agents in stem cell nutrient medium, and it could be a potentially biological material for neural tissue engineering (Figure 6) [42]. It was demonstrated that human neural stem cells seeded on hybrid scaffold of graphene nanoparticles exhibited a distinctive behavior that the neurite of differentiated neural stem cells showed increased growth and arrangement. Furthermore, it was demonstrated that the orientation of axons was largely owing to graphene and the radical nanometer particle monostratum leading to the increased differentiation of human neural stem cells into neuron which could be potentially used in nerve tissue engineering [55]. It was reported that fluorinated graphene accelerated the differentiation of MSCs into neuron lines; furthermore, cell alignment caused by fluorinated graphene with typographic dimethyl silicone polymer tunnel arrays obviously strengthened MSCs neuroinduction without any chemic inductors [56].

5.3.2. Three-Dimensional Graphene. Neural regeneration is a promising method for nerve injury repair with neural stem cells in clinical, for which 3D nanostructure scaffolds are necessary to offer culture microhabitats and collaboratively proportionate direction cues for the stem cells. A novel $3 \mathrm{D}$ poriferous scaffold was manufactured by graphene foam used for neural stem cell culture. It was demonstrated that the $3 \mathrm{D}$ graphene foam could sustain the growth of stem cell cultured on it as compared with 2D nanostructure, and the $3 \mathrm{D}$ graphene foam could maintain the proliferation of neural stem cell by promoting the gene expression of Ki67. At the same time, it was found that neural stem cell differentiated to stellate cells and particularly neurons by phenotype essay. Meanwhile, it was demonstrated that there was a superior electric coupling between stem cells which was differentiated with 3D nanostructure of graphene foams for effectual electrostimulation. These results indicated that 3D graphene foams might provide a novel terrace for nerve regeneration medicine [57].

5.3.3. Graphene Oxide. Nanoparticles are more likely to pass through cell membrane which is used as an ideal platform with biocompatibility and mechanically stability in regulating stem cell biological behavior. Transplant comprised of nanostructure biological material and stem cells is considered to be a clinical treatment in various neurological diseases. In light of recent developments, it was demonstrated that artificial synthesis of biological material based on carbon including carbon nanotubes and graphene could be used for enhancing the adhesion and differentiation of stem cell. 


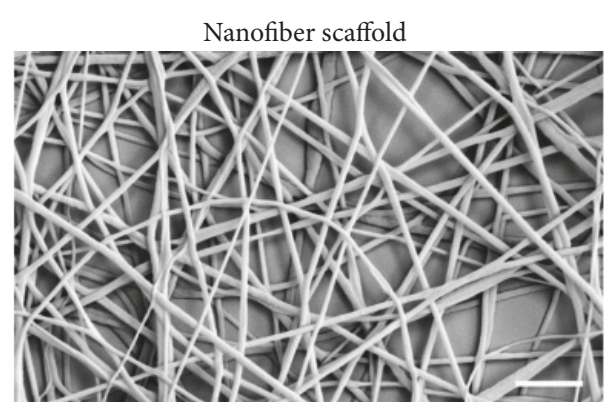

(a)

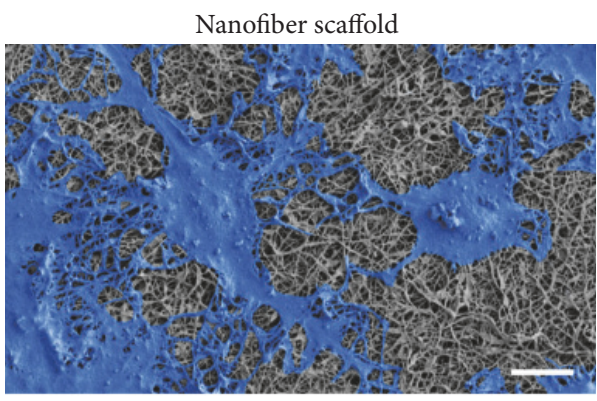

(c)

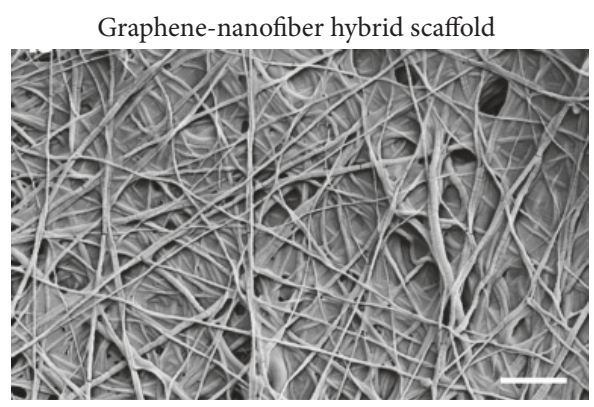

(b)

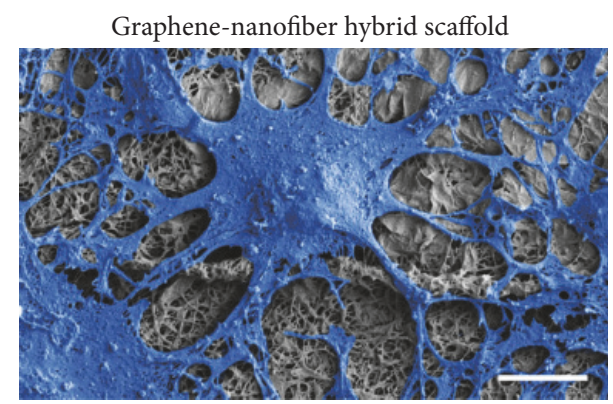

(d)

FIGURE 6: The morphology of nanofibrous scaffolds and cultured NSCs on the scaffolds. FE-SEM images of nanofibers (a) and graphenenanofibers hybrid scaffolds (b). FE-SEM of differentiated NSCs cultured on nanofiber scaffolds (c) and graphene-nanofiber hybrid scaffolds (d). The cells are pseudocolored blue for contrast. The differentiated cells on the grapheme-nanofiber hybrid scaffolds (d) show a clear morphological difference in terms of process extension compared with the nanofiber scaffolds alone (c). Figure 6 is from [42].

A research was carried out to investigate the function of different kinds of carbon nanomaterials on biological behaviors of dopamine nerval stem cells including graphene, graphene oxide, and carbon nanotubes. The results showed that merely graphene oxide efficaciously advanced the differentiation of dopamine neurons and the expression of correlative gene, and it indicated that graphene oxide could be promisingly used for neural stem cell therapy [58].

5.3.4. Mechanism of Graphene Biological Function. One of the main problems in nerve tissue regeneration is the development of biomaterials with powerful functions for managing the behavior of a particular cell and forming useful neural net. Surface topology structure of nanomaterial substrates provides opportunity to regulating the neural stem cell physiologic functions on molecular level. Grapheme is considered as a towardly candidate for nerval surfaces because of the unique properties of it in electrochemistry and machinery, while there is seldom understanding in the neural net structures on graphene as a biomaterial for nanostructures in regenerative medicine. Microarray research was carried out to explain the possible mechanism for the enhancement of human neural stem cell attachment and differentiation into neurons on grapheme substrate, and it was demonstrated that graphene electrodes can enhance differentiation of stem cells by electrical stimulation (Figure 7) [59]. The change in neural net combination of neural stem cell when cultured on graphene scaffold surface was investigated, and the results showed that graphene improved the serviceable neural circuits construction and enhanced the nerval behavior and electrophysiological signal of neural net which provided a further comprehension of interactions between neural stem cells with nanobiomaterial surface of grapheme, so it pointed out the great possibility of graphene as nerval separatrix in nerve tissue regeneration medicine [60].

\section{Conclusion}

In summary, stem cell research based on nanotechnology has obtained a lot of achievement in the field of regenerative medicine of orthopedic surgery, showing good prospects in clinical applications. But nanotechnology applied to stem cell research is still in its early stages, and there are a number of key issues to be solved. The objective of tissue engineering research with stem cell is to develop a system to accurately simulate the in vivo microenvironment system in vitro; however, the mechanism is not very clear regarding how nanomaterials similar to extracellular matrix in morphology affect the function of stem cells.

Biomaterials and their scaffold used for tissue engineering in orthopedic surgery can be manufactured by nanohydroxyapatite, titanium, calcium phosphate, graphene oxide, carbon nanotubes, or the compound of them. These composite scaffolds have both nanomaterials properties and good biocompatibility and low toxicity which render it a very broad application prospect in orthopedic surgery regenerative medicine by some nanoscale surface topography or molecular biology mechanism, including the regulation of 


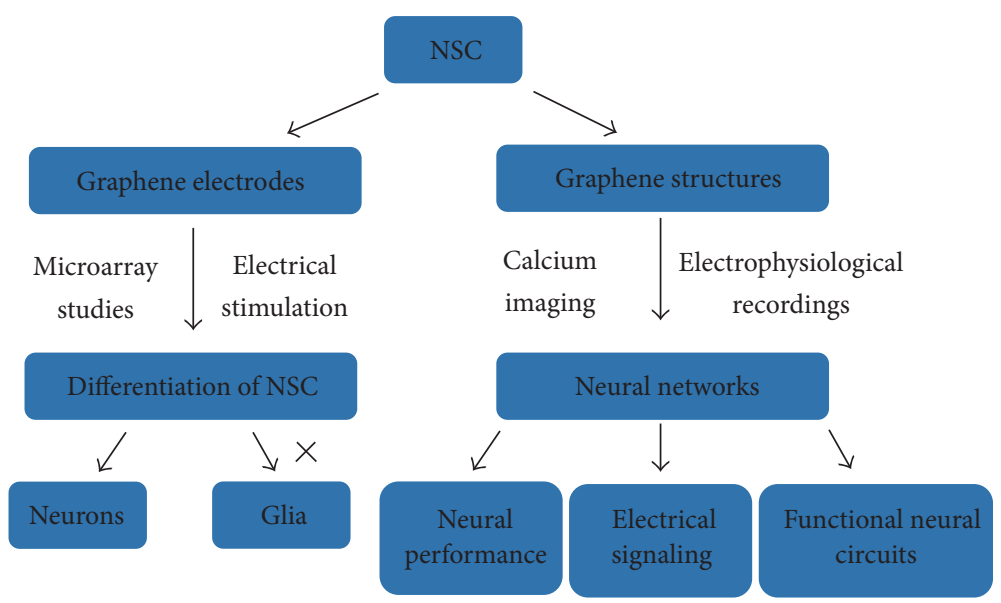

FIgURE 7: The mechanism of graphene biological function to NSCs.

stem cell biological behavior (such as attachment, proliferation, and differentiation), the improvement of bone tissue regeneration promising skeletal defects repair, internal fixation surgery, and spinal fusion in orthopedic surgery, the potential application in cartilage tissue engineering and recovery at tendon-to-bone insertion site, and the nerve tissue engineering for spinal cord injury.

Although the research of stem cell nanotechnology is facing many difficulties in regenerative medicine of orthopedic surgery, its potential applications have caused great concern. With the development of nanotechnology and stem cell research, the investigation of stem cell based on nanotechnology will be studied more in depth and will provide new methods for stem cell therapy and tissue engineering in regenerative medicine of orthopedic surgery.

\section{Conflicts of Interest}

The authors declare that there are no conflicts of interest regarding the publication of this paper.

\section{Acknowledgments}

This work was supported by the National Natural Science Foundation of China (Grant nos. 81301289 and 8167090834), the Youth Research Fund Project of Jilin Province Science and Technology Development Plan (Grant nos. 20130522032JH and 20130522039JH), and the Projects of International Cooperation of Jilin Provincial Science \& Technology Department (Grant no. 20150101175JC).

\section{References}

[1] Z. Yang, J. Xie, J. Zhu et al., "Functional exosome-mimic for delivery of siRNA to cancer: in vitro and in vivo evaluation," Journal of Controlled Release, vol. 243, pp. 160-171, 2016.

[2] J. Xie, Z. Yang, C. Zhou, J. Zhu, R. J. Lee, and L. Teng, "Nanotechnology for the delivery of phytochemicals in cancer therapy," Biotechnology Advances, vol. 34, no. 4, pp. 343-353, 2016.
[3] Z. Chen, Z. Chen, A. Zhang, J. Hu, X. Wang, and Z. Yang, "Electrospun nanofibers for cancer diagnosis and therapy," Biomaterials Science, vol. 4, no. 6, pp. 922-932, 2016.

[4] L. J. Lee, Z. Yang, M. Rahman et al., "Extracellular mRNA detected by tethered lipoplex nanoparticle biochip for lung adenocarcinoma detection," American Journal of Respiratory and Critical Care Medicine, vol. 193, no. 12, pp. 1431-1433, 2016.

[5] C. Kang, Y. Sun, J. Zhu et al., "Delivery of nanoparticles for treatment of brain tumor," Current Drug Metabolism, vol. 17, no. 8, pp. 745-754, 2016.

[6] Z. Yang, L. Chang, C.-L. Chiang, and L. J. Lee, "Micro-/nanoelectroporation for active gene delivery," Current Pharmaceutical Design, vol. 21, no. 42, pp. 6081-6088, 2015.

[7] Z. Yang, B. Yu, J. Zhu et al., "A microfluidic method to synthesize transferrin-lipid nanoparticles loaded with siRNA LOR-1284 for therapy of acute myeloid leukemia," Nanoscale, vol. 6, no. 16, pp. 9742-9751, 2014.

[8] C. Zhou, Z. Yang, and L. Teng, "Nanomedicine based on nucleic acids: pharmacokinetic and pharmacodynamic perspectives," Current Pharmaceutical Biotechnology, vol. 15, no. 9, pp. 829838, 2014.

[9] J. Xie, L. Teng, Z. Yang et al., "A polyethylenimine-linoleic acid conjugate for antisense oligonucleotide delivery," BioMed Research International, vol. 2013, Article ID 710502, 7 pages, 2013.

[10] Z. Chena, A. Zhang, Z. Yang et al., "Application of DODMA and derivatives in cationic nanocarriers for gene delivery," Current Organic Chemistry, vol. 20, no. 17, pp. 1813-1819, 2016.

[11] Z. Chen, M. Cong, J. Hu, Z. Yang, and Z. Chen, "Preparation of functionalized $\mathrm{TiO}_{2}$ nanotube arrays and their applications," Science of Advanced Materials, vol. 8, no. 6, pp. 1231-1241, 2016.

[12] X. Liu, D. Wei, J. Zhong et al., "Electrospun nanofibrous $\mathrm{P}$ (DLLA-CL) balloons as calcium phosphate cement filled containers for bone repair: in vitro and in vivo studies," ACS Applied Materials and Interfaces, vol. 7, no. 33, pp. 18540-18552, 2015.

[13] N. Xu, X. Ye, D. Wei et al., “3D artificial bones for bone repair prepared by computed tomography-guided fused deposition modeling for bone repair," ACS Applied Materials and Interfaces, vol. 6, no. 17, pp. 14952-14963, 2014.

[14] G. Sun, D. Wei, X. Liu et al., "Novel biodegradable electrospun nanofibrous $\mathrm{P}(\mathrm{DLLA}-\mathrm{CL})$ balloons for the treatment of 
vertebral compression fractures," Nanomedicine: Nanotechnology, Biology, and Medicine, vol. 9, no. 6, pp. 829-838, 2013.

[15] N. Panda, A. Bissoyi, K. Pramanik, and A. Biswas, "Directing osteogenesis of stem cells with hydroxyapatite precipitated electrospun eri-tasar silk fibroin nanofibrous scaffold," Journal of Biomaterials Science, Polymer Edition, vol. 25, no. 13, pp. 1440-1457, 2014.

[16] H. R. A. Balaji Raghavendran, S. Puvaneswary, S. Talebian et al., "A comparative study on in vitro osteogenic priming potential of electron spun scaffold PLLA/HA/Col, PLLA/HA, and PLLA/Col for tissue engineering application," PLOS ONE, vol. 9, no. 8, Article ID e104389, 2014.

[17] J. Hu, Y. Zhou, L. Huang, J. Liu, and H. Lu, "Effect of nanohydroxyapatite coating on the osteoinductivity of porous biphasic calcium phosphate ceramics," BMC Musculoskeletal Disorders, vol. 15, no. 1, article 114, 2014.

[18] Q. Lv, M. Deng, B. D. Ulery, L. S. Nair, and C. T. Laurencin, "Nano-ceramic composite scaffolds for bioreactor-based bone engineering basic research," Clinical Orthopaedics and Related Research, vol. 471, no. 8, pp. 2422-2433, 2013.

[19] A. Polini, D. Pisignano, M. Parodi, R. Quarto, and S. Scaglione, "Osteoinduction of human mesenchymal stem cells by bioactive composite scaffolds without supplemental osteogenic growth factors," PLoS ONE, vol. 6, no. 10, Article ID e26211, 2011.

[20] A. Khadka, J. Li, Y. Li, Y. Gao, Y. Zuo, and Y. Ma, "Evaluation of hybrid porous biomimetic nano-hydroxyapatite/polyamide 6 and bone marrow-derived stem cell construct in repair of calvarial critical size defect," Journal of Craniofacial Surgery, vol. 22, no. 5, pp. 1852-1858, 2011.

[21] B.-N. Lin, S. W. Whu, C.-H. Chen et al., "Bone marrow mesenchymal stem cells, platelet-rich plasma and nanohydroxyapatite-type I collagen beads were integral parts of biomimetic bone substitutes for bone regeneration," Journal of Tissue Engineering and Regenerative Medicine, vol. 7, no. 11, pp. 841-854, 2013.

[22] X. Liu, X. Li, Y. Fan et al., "Repairing goat tibia segmental bone defect using scaffold cultured with mesenchymal stem cells," Journal of Biomedical Materials Research Part B: Applied Biomaterials, vol. 94, no. 1, pp. 44-52, 2010.

[23] B. Qiao, J. Li, Q. Zhu et al., "Bone plate composed of a ternary nano-hydroxyapatite/polyamide 66/glass fiber composite: biomechanical properties and biocompatibility," International Journal of Nanomedicine, vol. 9, no. 1, pp. 1423-1432, 2014.

[24] Z.-B. Tang, J.-K. Cao, N. Wen et al., "Posterolateral spinal fusion with nano-hydroxyapatite-collagen/PLA composite and autologous adipose-derived mesenchymal stem cells in a rabbit model," Journal of Tissue Engineering and Regenerative Medicine, vol. 6, no. 4, pp. 325-336, 2012.

[25] P. J. ter Brugge, R. Torensma, J. E. De Ruijter, C. G. Figdor, and J. A. Jansen, "Modulation of integrin expression on rat bone marrow cells by substrates with different surface characteristics," Tissue Engineering, vol. 8, no. 4, pp. 615-626, 2002.

[26] S. Tetè, F. Mastrangelo, R. Quaresima et al., "Influence of novel nano-titanium implant surface on human osteoblast behavior and growth," Implant Dentistry, vol. 19, no. 6, pp. 520-531, 2010.

[27] M. Annunziata, A. Oliva, A. Buosciolo, M. Giordano, A. Guida, and L. Guida, "Bone marrow mesenchymal stem cell response to nano-structured oxidized and turned titanium surfaces," Clinical Oral Implants Research, vol. 23, no. 6, pp. 733-740, 2012.

[28] D. Khang, J. Choi, Y.-M. Im et al., "Role of subnano-, nanoand submicron-surface features on osteoblast differentiation of bone marrow mesenchymal stem cells," Biomaterials, vol. 33, no. 26, pp. 5997-6007, 2012.
[29] L. Lv, Y. Liu, P. Zhang et al., "The nanoscale geometry of $\mathrm{TiO} 2$ nanotubes influences the osteogenic differentiation of human adipose-derived stem cells by modulating $\mathrm{H} 3 \mathrm{~K} 4$ trimethylation," Biomaterials, vol. 39, pp. 193-205, 2015.

[30] M. Wang, N. J. Castro, J. Li, M. Keidar, and L. G. Zhang, "Greater osteoblast and mesenchymal stem cell adhesion and proliferation on titanium with hydrothermally treated nanocrystalline hydroxyapatite/magnetically treated carbon nanotubes," Journal of Nanoscience and Nanotechnology, vol. 12, no. 10, pp. 76927702, 2012.

[31] M. B. Eslaminejad, M. Zandi, E. Nejati, and E. Zomorodian, "Study of mesenchymal stem cell proliferation and bone differentiation on composite scaffolds of PLLA and nano hydroxyapatite with different morphologies," Cell Journal, vol. 12, no. 4, pp. 469-476, 2010.

[32] E. García-Gareta, J. Hua, J. C. Knowles, and G. W. Blunn, "Comparison of mesenchymal stem cell proliferation and differentiation between biomimetic and electrochemical coatings on different topographic surfaces," Journal of Materials Science: Materials in Medicine, vol. 24, no. 1, pp. 199-210, 2013.

[33] Y. C. Chai, G. Kerckhofs, S. J. Roberts et al., "Ectopic bone formation by 3D porous calcium phosphate-Ti6Al4V hybrids produced by perfusion electrodeposition," Biomaterials, vol. 33, no. 16, pp. 4044-4058, 2012.

[34] H. H. K. Xu, L. Zhao, M. S. Detamore, S. Takagi, and L. C. Chow, "Umbilical cord stem cell seeding on fast-resorbable calcium phosphate bone cement," Tissue Engineering-Part A, vol. 16, no. 9, pp. 2743-2753, 2010.

[35] J. L. Moreau and H. H. K. Xu, "Mesenchymal stem cell proliferation and differentiation on an injectable calcium phosphate-chitosan composite scaffold," Biomaterials, vol. 30, no. 14, pp. 2675-2682, 2009.

[36] T. R. Nayak, H. Andersen, V. S. Makam et al., "Graphene for controlled and accelerated osteogenic differentiation of human mesenchymal stem cells," ACS Nano, vol. 5, no. 6, pp. 46704678, 2011.

[37] S. Kang, J. B. Park, T.-J. Lee et al., "Covalent conjugation of mechanically stiff graphene oxide flakes to three-dimensional collagen scaffolds for osteogenic differentiation of human mesenchymal stem cells," Carbon, vol. 83, pp. 162-172, 2015.

[38] S. Kumar, S. Raj, E. Kolanthai, A. K. Sood, S. Sampath, and K. Chatterjee, "Chemical functionalization of graphene to augment stem cell osteogenesis and inhibit biofilm formation on polymer composites for orthopedic applications," ACS Applied Materials and Interfaces, vol. 7, no. 5, pp. 3237-3252, 2015.

[39] G. Marletta, G. Ciapetti, C. Satriano, F. Perut, M. Salerno, and N. Baldini, "Improved osteogenic differentiation of human marrow stromal cells cultured on ion-induced chemically struc-

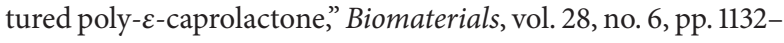
1140, 2007.

[40] D. Sankar, K. T. Shalumon, K. P. Chennazhi, D. Menon, and R. Jayakumar, "Surface plasma treatment of poly(caprolactone) micro, nano, and multiscale fibrous scaffolds for enhanced osteoconductivity," Tissue Engineering-Part A, vol. 20, no. 1112, pp. 1689-1702, 2014.

[41] C. Cunha, S. Panseri, O. Villa, D. Silva, and F. Gelain, "3D culture of adult mouse neural stem cells within functionalized self-assembling peptide scaffolds," International Journal of Nanomedicine, vol. 6, pp. 943-955, 2011.

[42] S. Shah, P. T. Yin, T. M. Uehara, S.-T. D. Chueng, L. Yang, and K.B. Lee, "Guiding stem cell differentiation into oligodendrocytes 
using graphene-nanofiber hybrid scaffolds," Advanced Materials, vol. 26, no. 22, pp. 3673-3680, 2014.

[43] A. Polini, S. Scaglione, R. Quarto, and D. Pisignano, "Composite electrospun nanofibers for influencing stem cell fate," in Stem Cell Nanotechnology: Methods and Protocols, K. Turksen, Ed., pp. 25-40, Humana Press, Totowa, NJ, USA, 2013.

[44] O. F. Zouani, C. Chanseau, B. Brouillaud et al., "Altered nanofeature size dictates stem cell differentiation," Journal of Cell Science, vol. 125, no. 5, pp. 1217-1224, 2012.

[45] M. Manfrini, C. Di Bona, A. Canella et al., "Mesenchymal stem cells from patients to assay bone graft substitutes," Journal of Cellular Physiology, vol. 228, no. 6, pp. 1229-1237, 2013.

[46] B. Holmes, N. J. Castro, J. Li, M. Keidar, and L. G. Zhang, "Enhanced human bone marrow mesenchymal stem cell functions in novel 3D cartilage scaffolds with hydrogen treated multi-walled carbon nanotubes," Nanotechnology, vol. 24, no. 36, Article ID 365102, 2013.

[47] D. Xue, Q. Zheng, C. Zong et al., "Osteochondral repair using porous poly(lactide-co-glycolide)/ nano-hydroxyapatite hybrid scaffolds with undifferentiated mesenchymal stem cells in a rat model," Journal of Biomedical Materials Research-Part A, vol. 94, no. 1, pp. 259-270, 2010.

[48] H. H. Yoon, S. H. Bhang, T. Kim, T. Yu, T. Hyeon, and B.-S. Kim, "Dual roles of graphene oxide in chondrogenic differentiation of adult stem cells: cell-adhesion substrate and growth factordelivery carrier," Advanced Functional Materials, vol. 24, no. 41, pp. 6455-6464, 2014.

[49] Y. Yang, J. Zhang, Y. Qian et al., "Superparamagnetic iron oxide is suitable to label tendon stem cells and track them in vivo with MR imaging," Annals of Biomedical Engineering, vol. 41, no. 10, pp. 2109-2119, 2013.

[50] G. J. Bakeine, J. Ban, G. Grenci et al., "Design, fabrication and evaluation of nanoscale surface topography as a tool in directing differentiation and organisation of embryonic stemcell-derived neural precursors," Microelectronic Engineering, vol. 86, no. 4-6, pp. 1435-1438, 2009.

[51] S. Irani, M. Zandi, N. Salamian, S. M. Saeed, M. Daliri Joupari, and S. M. Atyabi, "The study of P19 stem cell behavior on aligned oriented electrospun poly(lactic-co-glycolic acid) nano-fibers for neural tissue engineering," Polymers for Advanced Technologies, vol. 25, no. 5, pp. 562-567, 2014.

[52] M. Navaei-Nigjeh, G. Amoabedini, A. Noroozi et al., "Enhancing neuronal growth from human endometrial stem cells derived neuron-like cells in three-dimensional fibrin gel for nerve tissue engineering," Journal of Biomedical Materials Research. Part A, vol. 102, no. 8, pp. 2533-2543, 2014.

[53] A. Rahjouei, S. Kiani, A. Zahabi, N. Z. Mehrjardi, M. Hashemi, and H. Baharvand, "Interactions of human embryonic stem cell-derived neural progenitors with an electrospun nanofibrillar surface in vitro," The International Journal of Artificial Organs, vol. 34, no. 7, pp. 559-570, 2011.

[54] S. Ryu and B.-S. Kim, "Culture of neural cells and stem cells on graphene," Tissue Engineering and Regenerative Medicine, vol. 10, no. 2, pp. 39-46, 2013.

[55] A. Solanki, S.-T. D. Chueng, P. T. Yin, R. Kappera, M. Chhowalla, and K.-B. Lee, "Axonal alignment and enhanced neuronal differentiation of neural stem cells on graphene-nanoparticle hybrid structures," Advanced Materials, vol. 25, no. 38, pp. 54775482, 2013.

[56] Y. Wang, W. C. Lee, K. K. Manga et al., "Fluorinated graphene for promoting neuro-induction of stem cells," Advanced Materials, vol. 24, no. 31, pp. 4285-4290, 2012.
[57] H. Yoon, S. H. Ahn, and G. H. Kim, “Three-dimensional polycaprolactone hierarchical scaffolds supplemented with natural biomaterials to enhance mesenchymal stem cell proliferation," Macromolecular Rapid Communications, vol. 30, no. 19, pp. 1632-1637, 2009.

[58] D. Yang, T. Li, M. Xu et al., "Graphene oxide promotes the differentiation of mouse embryonic stem cells to dopamine neurons," Nanomedicine, vol. 9, no. 16, pp. 2445-2455, 2014.

[59] S. Y. Park, J. Park, S. H. Sim et al., "Enhanced differentiation of human neural stem cells into neurons on graphene," Advanced Materials, vol. 23, no. 36, pp. H263-H267, 2011.

[60] M. Tang, Q. Song, N. Li, Z. Jiang, R. Huang, and G. Cheng, "Enhancement of electrical signaling in neural networks on graphene films," Biomaterials, vol. 34, no. 27, pp. 6402-6411, 2013. 

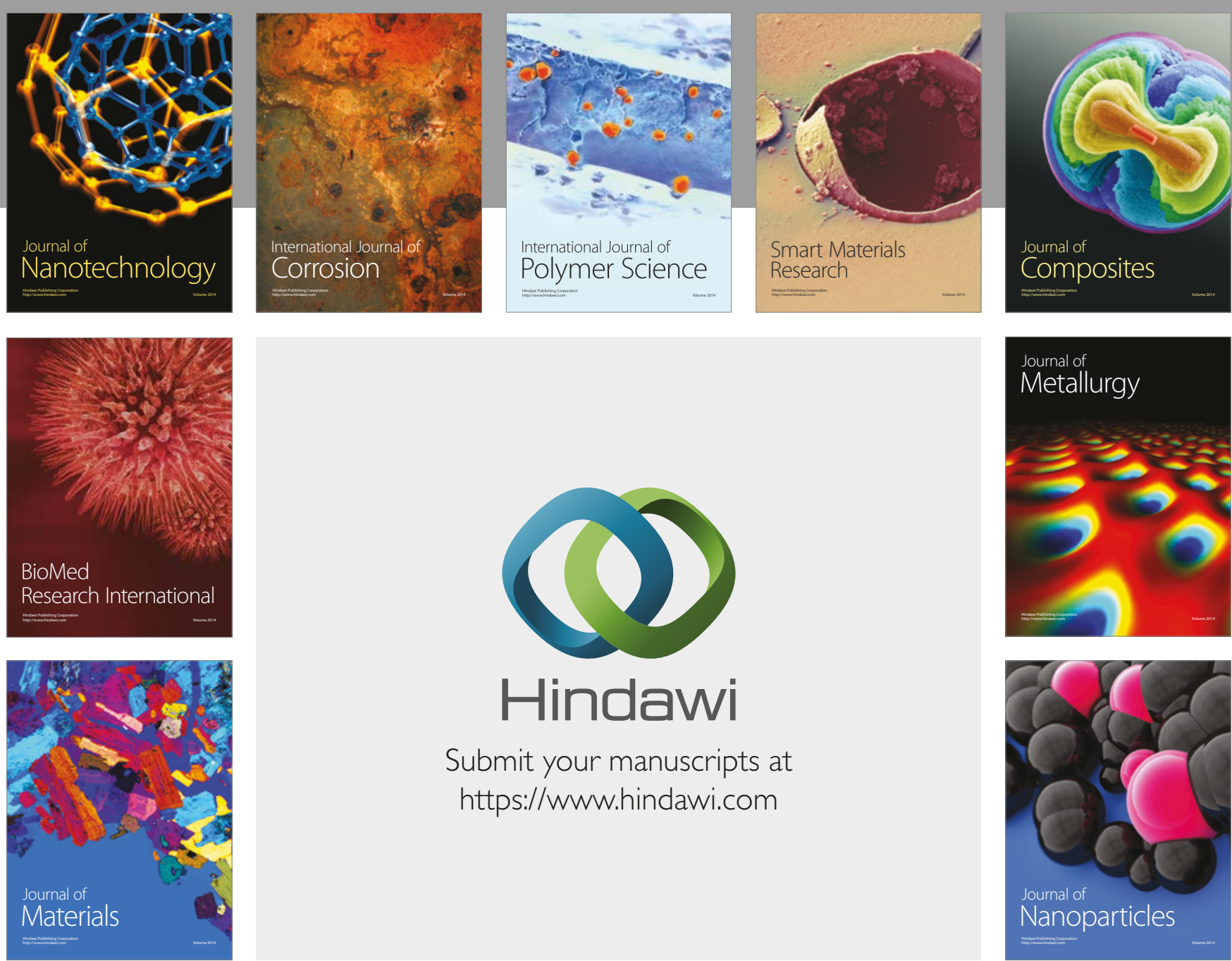

\section{Hindawi}

Submit your manuscripts at

https://www.hindawi.com
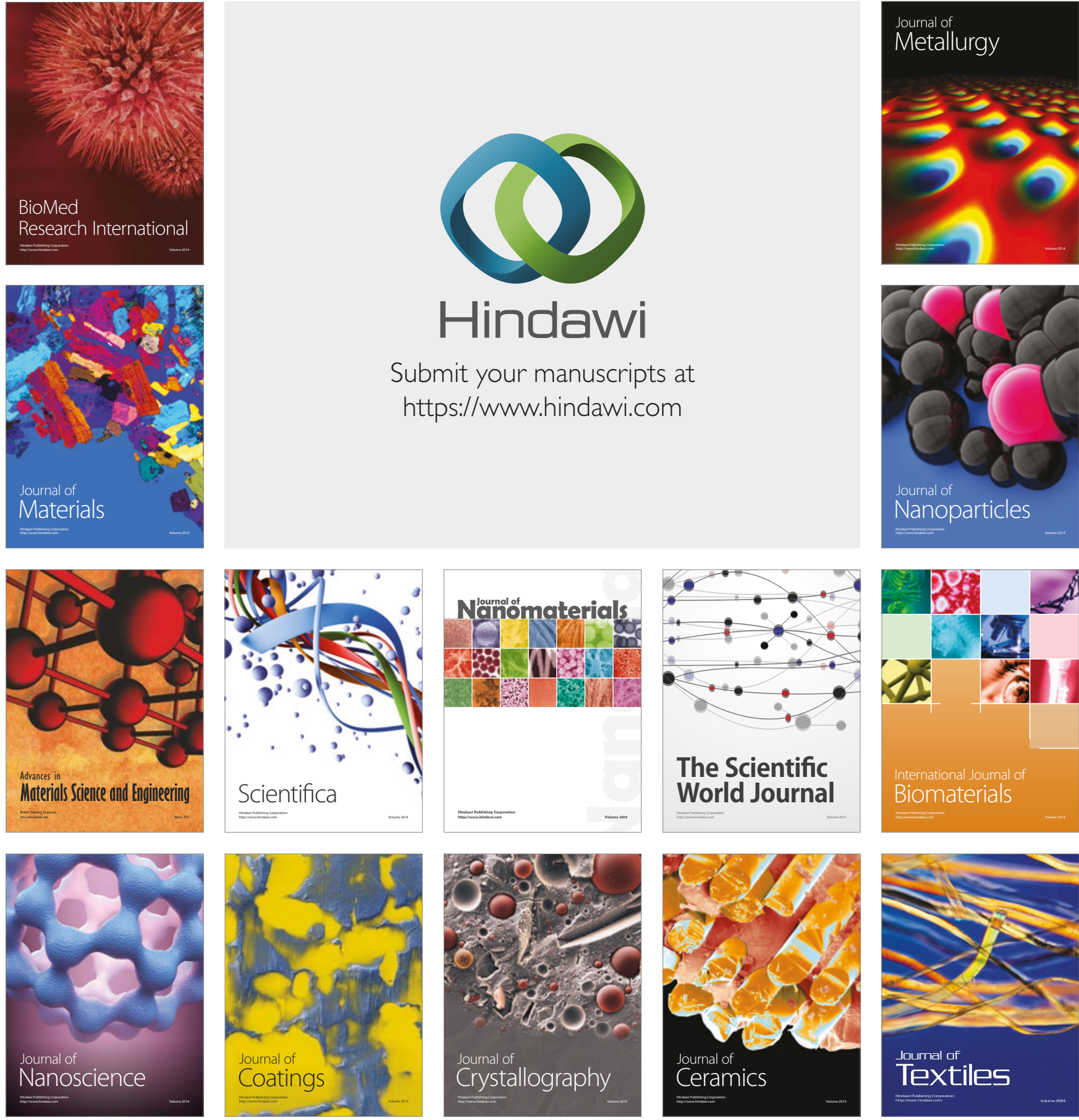

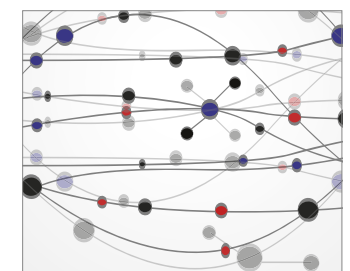

The Scientific World Journal
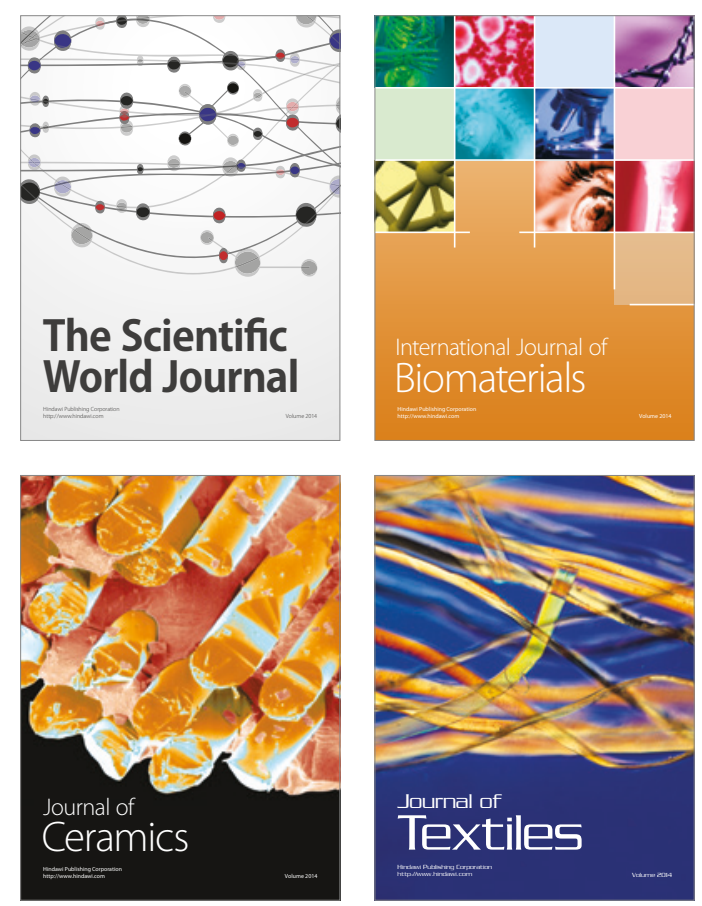